\title{
Bridging the Imaging Gap: PSMA PET/CT Has a High Impact on Treatment Planning in Prostate Cancer Patients with Biochemical Recurrence-A Narrative Review of the Literature
}

\author{
Özgül Ekmekcioglu ${ }^{1}$, Martijn Busstra ${ }^{2}$, Natalie Desiree Klass ${ }^{3}$, and Fred Verzijlbergen ${ }^{4}$ \\ ${ }^{I}$ Nuclear Medicine Department, Sisli Etfal Education and Research Hospital, Istanbul, Turkey; ${ }^{2}$ Urology Department, Erasmus \\ Medical Center, Rotterdam, The Netherlands; ${ }^{3}$ Radiation Oncology Department, Bern University Hospital, Bern, Switzerland; and \\ ${ }^{4}$ Radiology and Nuclear Medicine Department, Radboud University Medical Center, Nijmegen, The Netherlands
}

\begin{abstract}
${ }^{68} \mathrm{Ga}$ - and ${ }^{18} \mathrm{~F}$-labeled prostate-specific membrane antigen (PSMA) molecules have created new opportunities for the unmet diagnostic needs in prostate cancer. The purpose of this article is to give an overview of studies that have examined the role of PSMA PET in treatment planning for prostate cancer patients with biochemical recurrence (BCR). Methods: Medline, Embase, Web of Science, Google Scholar, and Cochrane Central were searched for relevant articles. After excluding the articles that did not fulfill the required criteria, we included in this review 12 publications that reported the impact of PSMA PET on the treatment plan for prostate cancer patients with BCR. Results: All studies in our review emphasized the impact of PSMA PET images on therapy management in prostate cancer patients with BCR. Overall, the impact of PSMA PET/CT on therapy management varied between $30 \%$ and $76 \%$ among the 1,346 patients included in the review. Upstaging was reported in $32 \%-67 \%$ of the patients. Patients with low prostate-specific antigen values $(<0.5 \mathrm{ng} / \mathrm{mL})$ also demonstrated positive lesions, which could not have been detected by means of conventional imaging techniques. Important modifications to the original treatment plan included avoidance of systemic therapy (17\%-40\%) and PET-directed local therapy (in $\leq 60 \%$ of the patients). Conclusion: PSMA imaging demonstrated a high clinical impact in patients with BCR, with modifications to the original treatment plan occurring among half the patients. Detecting recurrence in BCR can prevent unnecessary toxicity and lead to individualized therapy.
\end{abstract}

Key Words: ${ }^{18} \mathrm{~F}-\mathrm{PSMA} ;{ }^{68} \mathrm{Ga}$ PSMA; prostate cancer; biochemical recurrence; therapy impact

J Nucl Med 2019; 60:1394-1398

DOI: 10.2967/jnumed.118.222885

\section{$\mathbf{P}$}

rostate cancer (PC) is the second most common malignancy in men worldwide, with an incidence of 1.1 million in 2012; it is also the fifth major cause of death in men, with an estimated cancer-related mortality rate of $6.6 \%$ (1). Although most low-risk

Received Nov. 3, 2018; revision accepted Mar. 4, 2019.

For correspondence or reprints contact: Özgül Ekmekcioglu, Sisli Etfal Education and Research Hospital, Nuclear Medicine Department, Halaskargazi Cad. Etfal Sk., 34371 Sisli, Istanbul, Turkey.

E-mail: ozgulek@gmail.com

Published online Mar. 8, 2019.

COPYRIGHT (C) 2019 by the Society of Nuclear Medicine and Molecular Imaging. prostate cancers remain indolent, high-risk cancers have low chances of cure and may become a menace to life. Correct diagnosis and treatment continue to pose challenges $(2,3)$. Considering the general increase in life expectancy, treatment-related toxicities can have a strong impact on patients' quality of life for many years. Thus, the performance status and life expectancy of each patient should be taken into consideration before a decision is made about treatment options. Therefore, reliable prognostic tools and precise diagnostic tools are needed.

Routinely applied imaging modalities are not sufficiently sensitive and specific to be able to detect minute prostate cancer lesions, with a sensitivity of only $57 \%$ for lymph node metastases of 5$6 \mathrm{~mm}$ (4). Multiparametric MRI might indicate false-positive results (5). However, there is growing evidence that the treatment of a limited number of small metastases (oligometastases) may increase the likelihood of metastasis-free survival and postpone palliative systemic treatments (e.g., androgen deprivation therapy [ADT]) (6-8).

In the past $3 \mathrm{y}$, prostate-specific membrane antigen (PSMA)guided imaging and therapies have shown impressive progress and have been rapidly implemented in clinical practice. Afshar-Oromieh et al. published a large-scale study $(n=1007)$ on patients with recurrent prostate cancer in which ${ }^{68} \mathrm{Ga}$-PMSA-11 PET/CT was able to detect lesions with a $79.5 \%$ patient-based sensitivity (9).

PSMA is a type II transmembrane protein that contributes to glutamatergic neurotransmission and folate absorption. Despite the name, PSMA is expressed not only in prostate but also in salivary and lacrimal glands, kidney, nervous system, duodenum, and colon (10-13). PSMA is expressed more intensely in prostate cancer cells than in normal prostate tissues and is related to increased folate activity in cells, giving them a proliferative advantage $(14,15)$. Therefore, PSMA has become a promising target for imaging and therapy in prostate cancer. Furthermore, the PSMA expression level in prostatectomy specimens has been shown to be an independent prognostic factor for disease outcome (16).

PSMA-targeting methods were first initiated with labeling of antibodies (Supplemental Fig. 1; supplemental materials are available at http://jnm.snmjournals.org). However, the resultant drawbacks of antibodies were overcome by developing small-molecule PSMA inhibitors. A variety of PSMA-directed ligands are used for either imaging or therapy (e.g., PSMA-617, PSMA-I\&T [imaging and therapy], and PSMA-11 [PSMA-HBED-CC]). PSMA-617, which can be labeled with ${ }^{177} \mathrm{Lu}$ and ${ }^{225} \mathrm{Ac}$, and PSMA I\&T were 
developed for radioligand therapy. Lately, ${ }^{18} \mathrm{~F}$-labeled PSMA ligands (e.g., PSMA-1007 and DCFPyL) have been developed, which can also be the solution to a higher image quality (17). Furthermore, ${ }^{18}$ F-fluciclovine (Axumin; Blue Earth Diagnostics, Inc.) is another useful, promising compound that is transported by a synthetic amino acid and has been approved by the Food and Drug Administration (18).

Biochemical recurrence (BCR) in prostate cancer means that prostate-specific antigen (PSA) in serum is rising without histologic or image-guided proof. BCR is generally considered the first sign of treatment failure. In most cases, especially with low PSA levels, conventional imaging techniques are not accurate enough to determine the location of recurrence. Recent studies into identifying the location of the metastases with ${ }^{68} \mathrm{Ga}$-PMSA PET/CT have changed the perspective of the therapy plan and the radiotherapy region and dose, especially in oligometastatic diseases.

We reviewed the articles on ${ }^{68} \mathrm{Ga}$ PSMA PET/CT among prostate cancer patients with BCR and the impact on modification of the treatment plan. Although several studies have recently been published on other ${ }^{18} \mathrm{~F}$-labeled agents, this study chose to concentrate on the effect of imaging on the therapy plan, which has received great attention using ${ }^{68} \mathrm{Ga}$-PSMA PET. We intended to summarize the benefits and shortcomings of ${ }^{68} \mathrm{Ga}$-PSMA PET and understand how current knowledge on PSMA imaging can be applied to clinical practice in a sensible and practical way.

\section{MATERIALS AND METHODS}

\section{Search Strategy}

Proper key words were used in June 2018 to systematically search the scientific databases of Medline, Embase, Web of Science, Google Scholar, and Cochrane Central for studies relating to ${ }^{68} \mathrm{Ga}$-PSMA $\mathrm{PET} / \mathrm{CT}$ imaging in prostate cancer with BCR. The search was conducted according to the search strategy and data collection guidelines of the Preferred Reporting Items for Systematic Reviews and MetaAnalyses statement (19).

\section{Data Collection and Extraction}

The abstracts of 859 articles were examined by 2 observers (Supplemental Fig. 2). Articles that contained information about ${ }^{68} \mathrm{Ga}-$ PSMA PET and data on patients with prostate cancer with BCR were included. Duplicate studies, letters to the editor, conference papers, case reports, preclinical studies, and non-English articles were excluded, as were studies on staging in PC and imaging with agents other than ${ }^{68} \mathrm{Ga}$-PSMA. Twelve articles matched the inclusion criteria. Differences of opinion between the reviewers were resolved through discussion.

\section{RESULTS}

Imaging protocols were specified in all 12 studies that reported ${ }^{68} \mathrm{Ga}$-PSMA PET findings for PC patients with BCR. Details were not given for 1 study (20); however, images of all other studies were interpreted by experienced nuclear medicine physicians and radiologists. PSMA lesions were characterized as positive if they demonstrated uptake above adjacent background and not attributable to physiologic biodistribution. Lesion locations were categorized as prostate bed, pelvic lymph nodes, extrapelvic retroperitoneal nodes, other lymph nodes, bone lesions, and viscera (21). When these studies were designed, no generally accepted guidelines were available. The studies are summarized in Supplemental Table 1.

Of the 12 articles identified, 7 were on retrospective studies and 5 were on prospective studies. Only 3 of the 5 prospective studies used questionnaires to collect data about the treatment plan before and after the results of PSMA imaging were known (22-24), with limited likelihood of bias in the results. The patients underwent initial evaluation for risk classification, TNM staging, and radiotherapy planning. Two of the 5 studies chose to follow the patients prospectively using the database $(20,25)$. The follow-up results provided useful insights into the effect of PSMA imaging on the intended therapy plan.

In their prospective study, Habl et al. conducted imaging using either ${ }^{68}$ Ga-PSMA PET/CT $(n=71)$ or ${ }^{68}$ Ga-PSMA PET/MRI $(n=29)$ after radical prostatectomy and before radiotherapy. ${ }^{68} \mathrm{Ga}$ PSMA PET was found to have a significant influence on TNM stage in $43 \%$ of the patients. The initial radiotherapy plan was adapted in $59 \%$ of the cases. Six patients who showed bone oligometastases and changed to M1b received stereotactic body radiotherapy (25).

Roach et al. asked the referring clinicians to record the therapy plan by completing a questionnaire before and after PSMA imaging. The results of $62 \%$ of the cases demonstrated a change in the treatment schedule. Even $67 \%$ of the patients with low PSA levels $(<0.2 \mathrm{ng} / \mathrm{mL})$ exhibited management changes, but quite unexpectedly, no significant difference was found between the PSA cohorts of the BCR patients. The PSMA images revealed either oligometastatic or polymetastatic disease in $76 \%$ of the patients, which resulted in a strong increase in systemic therapies, including ADT (22).

The survey conducted by Calais et al. documented a $53 \%$ rate of change in treatment. Specifically, there was a significant relationship between either N1 or N1M1 status in PSMA findings and management changes (23). In the study of Frenzel et al., when ${ }^{68} \mathrm{Ga}$ PSMA PET/CT images were retrospectively compared with CT alone, a change rate of $43 \%$ was observed. One specific patient was identified with a very low PSA level $(0.02 \mathrm{ng} / \mathrm{mL})$ and microscopic residual disease. In this instance, a decision was made not to prescribe radiotherapy, mainly because of the negative findings on PSMA images (26).

Considering the cardiovascular disadvantages and impact on the quality of life, $77 \%$ of a subgroup of patients with a preimaging decision to give ADT were withdrawn from ADT after ${ }^{68} \mathrm{Ga}-$ PSMA imaging (27). More than half the patients (i.e., 14/22) who were planned to receive ADT before PSMA imaging received locoregional treatment instead, and 1 patient did not even receive any treatment at all (28). The decision to start with ADT was also reconsidered quite frequently (64\%) in the study of Albisinni et al. Furthermore, other modifications included changes in the treatment plan concerning clinical surveillance $(22 \%)$, stereotactic radiotherapy (12\%), and salvage radiotherapy (11\%) (29).

The impact of ${ }^{68} \mathrm{Ga}$-PSMA imaging on the treatment plan has been emphasized in the prospective study by Shakespeare. The overall revision of treatment plan was $53.7 \%$, and radical radiotherapy appeared less promising after PSMA imaging. Moreover, instead of radical radiotherapy, palliative radiotherapy of oligometastatic disease was implemented. Additionally, the number of patients $(50 \%)$ without any treatment decision significantly diminished (18.5\%) after PSMA imaging (20).

Sterzing et al. observed a $50.8 \%$ change in radiotherapeutic management after PSMA imaging. The treatment plan was changed to an additional integrated lymph node boost, enlarged lymphatic field irradiation, and systemic therapy arising from a change from $\mathrm{N} 0$ to $\mathrm{N} 1$ in $62.1 \%, \mathrm{M} 0$ to M1a in $27.5 \%$, and M0 to M1b in $13.8 \%$ among $29(50.8 \%)$ patients (30). Bluemel et al. and Farolfi et al. have also recommended a change in treatment rates based on the 
findings of ${ }^{68} \mathrm{Ga}$ PSMA PET/CT scans (i.e., $42.2 \%$ and $30.2 \%$, respectively) $(31,32)$.

In the study by Hope et al., a 53\% therapeutic change was highlighted after ${ }^{68} \mathrm{Ga}$ PSMA scanning. This study emphasized that $31.7 \%$ of the patients received focal targeted therapy instead of systemic therapy, and the intended treatments were affected by $59.6 \%$ overall (of which $53.2 \%$ were major changes) (24). Grubmüller et al. reported a detection rate of $85.5 \%$ of PSMApositive lesions in a patient group with BCR. The therapeutic strategy was changed among $74.6 \%$ (50/67) of the patients. Furthermore, $60 \%$ of the patients received PET-guided radiotherapy (33).

Here, all available data on PSMA imaging in patients with BCR are summarized, although not all studies in our review reported the modification rates identically. Overall, the impact of ${ }^{68} \mathrm{Ga}$ PSMA PET/CT on therapy management varied between $30.2 \%$ and $76 \%$ among the patients with BCR. Furthermore, the number of upstaged patients was in the range of $32 \%-67 \%$, which was also remarkable.

Among $60 \%$ of the patients, the main modifications consisted of avoidance of systemic therapy (17\%-40\%) and PET-directed local therapy, which includes radiotherapy to the prostatic or pelvic bed $(7.5 \%-48.7 \%)$ or treatment of oligometastatic disease $(10 \%-28 \%)$. The results from PSMA-enhanced adaptations of the radiotherapy field were as follows: stereotactic body radiotherapy, 10\%-50\%; simultaneous integrated boost to lymph nodes, $15 \%-62.1 \%$; and salvage radiotherapy, $11 \%-24.4 \%$. The avoidance in ADT treatment was emphasized in $33.3 \%-64 \%$ of the studies that specified these data separately. Changing the treatment plan in active surveillance was mentioned in $2.5 \%-22 \%$ of cases, and lymphadenectomy after PSMA rates were reported as not negligible (1\%-13\%).

The studies in our review had a wide range of PSA valuesfrom 0.01 to $653.2 \mathrm{ng} / \mathrm{mL}$. Despite the low PSA values, Habl et al. found that nearly $50 \%$ of the patients with a PSA level of less than $0.5 \mathrm{ng} / \mathrm{mL}$ had a modified therapy plan after PSMA imaging (25). Additionally, Roach et al. mentioned no significant relationship between therapy management and PSA values (26). Moreover, Sterzing et al. documented 16 PSMA-negative patients in the $0.5-21.7 \mathrm{ng} / \mathrm{mL}$ PSA range (30). On the other hand, positive rates between $21 \%$ and $65 \%$ were reported for patients with a PSA level of less than $0.5 \mathrm{ng} / \mathrm{mL}$ in 5 of 12 studies in our review. Finally, it should be noted that low PSA values with a low risk of systemic disease have a positive effect on therapy management, and vice versa.

\section{DISCUSSION}

This review aimed to summarize 12 studies on the effect of PSMA PET/CT on treatment perspective and outcome among BCR patients. Of the 12 studies, only 5 were prospective. Other important drawbacks included the small size of the study groups and lack of long-term outcomes and validation of histology. Only 2 of the studies have evaluated a group of patients by using PET/MRI $(25,33)$.

PSA should be less than $0.1 \mu \mathrm{g} / \mathrm{L}$ after surgical treatment and radiotherapy (34). A measurable and rising PSA level after prostatectomy or a PSA level greater than $2.0 \mu \mathrm{g} / \mathrm{L}$ above nadir is referred to as a biochemical prostate cancer recurrence (BCR). In BCR, salvage treatments can be offered. Moreover, several studies have shown that salvage radiotherapy is most effective at PSA levels of less than $0.5 \mu \mathrm{g} / \mathrm{L}(34,35)$. But at this PSA range, traditional imaging will not be able to locate recurrence; therefore, salvage treatments were directed at the region with the highest chance of recurrence (i.e., prostatic fossa).
Knowing that locoregional salvage therapies are most effective at PSA levels of less than $0.5 \mu \mathrm{g} / \mathrm{L}$, it became evident that more sensitive imaging is essential to avoid the unnecessary toxicity of treatments. A recent metaanalysis with 1,052 patients showed an overall positive ${ }^{68} \mathrm{Ga}$-PSMA PET/CT detection rate of $76 \%$ among the PC patients with BCR (36). In another study, the efficacy of the ${ }^{68} \mathrm{Ga}$ PSMA PET/CT detection rate was $58 \%$ among patients with a PSA level of less than $0.5 \mathrm{ng} / \mathrm{mL}$; it was also deemed important as it is the upper limit for salvage radiation therapy, as determined by the European Association of Urology guidelines (37). Furthermore, a greater detection efficacy of $81.8 \%$ was found among another group of BCR patients with PSA values between 2 and $>5 \mathrm{ng} / \mathrm{mL}$ (38). To date, PSMA-based imaging has proven to be the most promising detection technique at low PSA levels $(30,32)$.

In patients with tumor recurrence outside the prostate bed, the possibility of an efficient salvage therapy depends on the number and localization of lesions (39). In cases with numerous or diffuse lesions, it is mainly antihormonal treatment or other systemic therapies that can be administered. ADT has even been proven to improve survival rates, decrease the quality of life arising from sexual dysfunction, and lead to cardiovascular disease and metabolic complications with negative effects on bone density and cognitive function $(27,40,41)$. However, growing evidence has recently shown that lowvolume metastatic disease can effectively be treated by local therapies (e.g., individualized selective pelvic irradiation). Because it has been shown that prostate cancer with initial low numbers of metastases is more likely to show local progression when treated with ADT, local metastasis-directed therapy can be considered a viable option $(42,43)$.

Preventing unnecessary radiotherapy or postponing palliative ADT can have a major effect on patients' quality of life. Such a goal can be achieved by PSMA imaging, which enables assessing the extent of disease and localizing oligometastatic disease or local recurrence in the prostate bed. There is also evidence of benefits arising from locoregional treatment of the oligometastatic prostate cancer $(44,45)$.

Considering the benefits of cyclotron production and longer half-life, ${ }^{18} \mathrm{~F}$ is a feasible PET agent. ${ }^{18} \mathrm{~F}$-PSMA PET imaging in prostate cancer has recently been introduced. In 2 separate studies ( $n=25$ and $n=14$ ), more lesions were detected on ${ }^{18} \mathrm{~F}$-PSMA PET images than on ${ }^{68} \mathrm{Ga}$-PSMA PET/CT images $(46,47)$. In their recently published study on the detection efficacy of ${ }^{18}$ F-PSMA1007 imaging in patient groups with BCR, Giesel et al. found that the detection rate was $61.5 \%$ among patients with PSA levels of 0.2 to less than $0.5 \mathrm{ng} / \mathrm{mL}$; they also reported that less excretion to the urinary tract was a clear advantage (48). Another study on ${ }^{18} \mathrm{~F}$ fluciclovine has reported a 59\% rate of change in the therapy management plan for patients with BCR. However, because the patients' outcomes were not available for this study, further prospective trials are required (49). In addition, when ${ }^{18} \mathrm{~F}-\mathrm{PSMA}$ (DCFPyL) images were compared with ${ }^{18} \mathrm{~F}$-fluciclovine for the same patient, it was possible to detect metastatic lesions in both studies. PSMA images demonstrated higher lesion uptake with lower background activity; however, low urinary excretion of ${ }^{18} \mathrm{~F}$-fluciclovine could give better results in demonstrating pelvic lesions (50).

A few studies have already shown the high impact and good response of PSMA-based therapy on outcome (51-53). However, limitations such as small samples of patients, exclusion of those with metastatic disease, and short-term follow-up might account for nonidentical categories of data. A prospective multicenter study demonstrated the clinical performance and patient-outcome results for ${ }^{18} \mathrm{~F}$-fluoromethylcholine, multiparametric MRI, and 
${ }^{68}$ Ga-PSMA PET/CT in BCR after 3 y of follow-up. The study reported high detection rates of disease, especially in extrapelvic fossae. Moreover, the impact on the therapy management plan and on treatment response to salvage radiotherapy was greater among the patient group with negative imaging results (54). Another recently published retrospective biinstitutional study, by SchmidtHegemann et al., has provided valuable insights about PET-based salvage radiotherapy in 90 patients with BCR after 23 mo of follow-up. The data emphasized a protracted treatment response as well as a low toxicity profile and a $78 \%$ BCR-free survival rate after ${ }^{68} \mathrm{Ga}$-PSMA imaging. The limitation of this retrospective analysis includes treatment and a nonidentical follow-up, which requires more prospective studies with larger cohorts (55).

The limitations of this review originate mainly in a lack of grouping of the results because of the availability of a variety of management plans in each study, which either were different or lacked sufficient detail. The results of the studies demonstrate that early and accurate detection of BCR or ruling out oligometastases will influence decisions concerning curative therapy and eventually change the outcome of the disease and the quality of life. More longitudinal studies are required to examine the outcome of the PSMA PET/CT imaging-guided therapy plan and modification.

\section{CONCLUSION}

${ }^{68} \mathrm{Ga}$-PSMA PET/CT scan has a $52.8 \%$ positive impact on therapy management in patients with BCR. Our achievements today in PSMA molecules are beyond what we could have imagined a decade ago; however, more prospective randomized trials are required to understand the impact on disease-free survival rates and treatment outcome. In addition to improving progression-free survival rates, we also need to avoid and postpone treatment toxicity.

\section{DISCLOSURE}

No potential conflict of interest relevant to this article was reported.

\section{KEY POINTS}

QUESTION: What is the impact on treatment planning of the ${ }^{68} \mathrm{Ga}-/ 18 \mathrm{~F}-\mathrm{PSMA}$ scan in prostate cancer patients with PSA recurrence after primary treatment?

PERTINENT FINDINGS: In 12 studies, including 1,346 patients, PSMA PET/CT scans resulted in modification of the originally planned treatment schedule in $30 \%-76 \%$ of the patients. Important modifications included avoidance of systemic therapy and PET-directed local therapy.

IMPLICATIONS FOR PATIENT CARE: In almost every patient with PSA recurrence after radical prostatectomy or radiation treatment, a PSMA PET/CT scan is strongly recommended to determine the next treatment phase.

\section{REFERENCES}

1. Ferlay J, Soerjomataram I, Dikshit R, et al. Cancer incidence and mortality worldwide: sources, methods and major patterns in GLOBOCAN 2012. Int J Cancer. 2015;136:E359-E386.

2. D'Amico AV, Whittington R, Malkowicz SB, et al. Biochemical outcome after radical prostatectomy, external beam radiation therapy, or interstitial radiation therapy for clinically localized prostate cancer. JAMA. 1998;280: 969-974.
3. Nguyen CT, Kattan MW. Formalized prediction of clinically significant prostate cancer: is it possible? Asian J Androl. 2012;14:349-354.

4. Jilg CA, Schultze-Seemann W, Drendel V, et al. Detection of lymph node metastasis in patients with nodal prostate cancer relapse using ${ }^{18} \mathrm{~F} /{ }^{11} \mathrm{C}$-choline positron emission tomography/computerized tomography. J Urol. 2014;192:103110.

5. Notley M, Yu J, Fulcher A, Turner MA, Cockrell CH, Nguyen D. Diagnosis of recurrent prostate cancer and its mimics at multiparametric prostate MRI. $\mathrm{Br} \mathrm{J}$ Radiol. 2015;88:20150362.

6. Ost P, Jereczek-Fossa BA, As NV, et al. Progression-free survival following stereotactic body radiotherapy for oligometastatic prostate cancer treatmentnaive recurrence: a multi-institutional analysis. Eur Urol. 2016;69:9-12.

7. Van Poppel H, De Meerleer G, Joniau S. Oligometastatic prostate cancer: metastases-directed therapy? Arab J Urol. 2016;14:179-182.

8. Habl G, Straube C, Schiller K, et al. Oligometastases from prostate cancer: local treatment with stereotactic body radiotherapy (SBRT). BMC Cancer. 2017; $17: 361$.

9. Afshar-Oromieh A, Holland-Letz T, Giesel FL, et al. Diagnostic performance of ${ }^{68}$ Ga-PSMA-11 (HBED-CC) PET/CT in patients with recurrent prostate cancer: evaluation in 1007 patients. Eur J Nucl Med Mol Imaging. 2017;44:1258-1268.

10. De Marzo AM, Meeker AK, Zha S, et al. Human prostate cancer precursors and pathobiology. Urology. 2003;62:55-62.

11. Zhao R, Matherly LH, Goldman ID. Membrane transporters and folate homeostasis: intestinal absorption and transport into systemic compartments and tissues. Expert Rev Mol Med. 2009;11:e4.

12. Shafizadeh TB, Halsted CH. Gamma-glutamyl hydrolase, not glutamate carboxypeptidase II, hydrolyzes dietary folate in rat small intestine. J Nutr. 2007; 137:1149-1153.

13. Afshar-Oromieh A, Malcher A, Eder M, et al. PET imaging with a $\left[{ }^{68} \mathrm{Ga}\right] \mathrm{gal}-$ lium-labelled PSMA ligand for the diagnosis of prostate cancer: biodistribution in humans and first evaluation of tumour lesions. Eur J Nucl Med Mol Imaging. 2013;40:486-495.

14. Yao V, Berkman CE, Choi JK, O'Keefe DS, Bacich DJ. Expression of prostatespecific membrane antigen (PSMA), increases cell folate uptake and proliferation and suggests a novel role for PSMA in the uptake of the non-polyglutamated folate, folic acid. Prostate. 2010;70:305-316.

15. Silver DA, Pellicer I, Fair WR, Heston WD, Cordon-Cardo C. Prostate-specific membrane antigen expression in normal and malignant human tissues. Clin Cancer Res. 1997;3:81-85.

16. Ross JS, Sheehan CE, Fisher HA, et al. Correlation of primary tumor prostatespecific membrane antigen expression with disease recurrence in prostate cancer. Clin Cancer Res. 2003;9:6357-6362.

17. Schwarzenboeck SM, Rauscher I, Bluemel C, et al. PSMA ligands for PET imaging of prostate cancer. J Nucl Med. 2017;58:1545-1552.

18. Bach-Gansmo T, Nanni C, Nieh PT, et al. Multisite experience of the safety, detection rate and diagnostic performance of fluciclovine $\left({ }^{18} \mathrm{~F}\right)$ positron emission tomography/computerized tomography imaging in the staging of biochemically recurrent prostate cancer. J Urol. 2017;197:676-683.

19. Liberati A, Altman DG, Tetzlaff J, et al. The PRISMA statement for reporting systematic reviews and meta-analyses of studies that evaluate healthcare interventions: explanation and elaboration. BMJ. 2009;339:b2700.

21. Fendler WP, Eiber M, Beheshti M, et al. ${ }^{68} \mathrm{Ga}$-PSMA PET/CT: joint EANM and SNMMI procedure guideline for prostate cancer imaging: version 1.0. Eur J Nucl Med Mol Imaging. 2017;44:1014-1024.

22. Roach PJ, Francis R, Emmett L, et al. The impact of ${ }^{68} \mathrm{Ga}-\mathrm{PSMA}$ PET/CT on management intent in prostate cancer: results of an Australian prospective multicenter study. J Nucl Med. 2018;59:82-88.

23. Calais J, Fendler WP, Eiber M, et al. Impact of ${ }^{68} \mathrm{Ga}$-PSMA-11 PET/CT on the management of prostate cancer patients with biochemical recurrence. $\mathrm{J} \mathrm{Nucl}$ Med. 2018;59:434-441.

24. Hope TA, Aggarwal R, Chee B. al. Impact of ${ }^{68}$ Ga-PSMA-11 PET on management in patients with biochemically recurrent prostate cancer. J Nucl Med. 2017; 58:1956-1961.

20. Shakespeare TP. Effect of prostate-specific membrane antigen positron emission tomography on the decision-making of radiation oncologists. Radiat Oncol. 2015; 10:233.

25. Habl G, Sauter K, Schiller K, et al. ${ }^{68} \mathrm{Ga}$-PSMA-PET for radiation treatment planning in prostate cancer recurrences after surgery: individualized medicine or new standard in salvage treatment. Prostate. 2017;77:920-927.

26. Frenzel T, Tienken M, Abel M, et al. The impact of $\left[{ }^{68} \mathrm{Ga}\right] P S M A ~ I \& T$ PET/CT on radiotherapy planning in patients with prostate cancer. Strahlenther Onkol. 2018;194:646-654

27. Roach M III, DeSilvio M, Lawton C, et al. Phase III trial comparing wholepelvic versus prostate-only radiotherapy and neoadjuvant versus adjuvant 
combined androgen suppression: Radiation Therapy Oncology Group 9413. J Clin Oncol. 2003;21:1904-1911.

28. De Bari B, Mazzola R, Aiello D, et al. Could 68-Ga PSMA PET/CT become a new tool in the decision-making strategy of prostate cancer patients with biochemical recurrence of PSA after radical prostatectomy? A preliminary, monocentric series. Radiol Med (Torino). 2018;123:719-725.

29. Albisinni S, Artigas C, Aoun F, et al. Clinical impact of ${ }^{68}$ Ga-prostate-specific membrane antigen (PSMA) positron emission tomography/computed tomography (PET/CT) in patients with prostate cancer with rising prostate-specific antigen after treatment with curative intent: preliminary analysis of a multidisciplinary approach. BJU Int. 2017;120:197-203.

30. Sterzing F, Kratochwil C, Fiedler H, et al. ${ }^{68} \mathrm{Ga}-\mathrm{PSMA}-11$ PET/CT: a new technique with high potential for the radiotherapeutic management of prostate cancer patients. Eur J Nucl Med Mol Imaging. 2016;43:34-41.

31. Bluemel C, Linke F, Herrmann $\mathrm{K}$, et al. Impact of ${ }^{68} \mathrm{Ga}$-PSMA PET/CT on salvage radiotherapy planning in patients with prostate cancer and persisting PSA values or biochemical relapse after prostatectomy. EJNMMI Res. 2016;6:78.

32. Farolfi A, Ceci F, Castellucci P, et al. ${ }^{68} \mathrm{Ga}$-PSMA-11 PET/CT in prostate cancer patients with biochemical recurrence after radical prostatectomy and PSA $<0.5$ ng/ml: efficacy and impact on treatment strategy. Eur J Nucl Med Mol Imaging. 2019;46:11-19.

33. Grubmüller B, Baltzer P, D'Andrea D, et al. ${ }^{68}$ Ga-PSMA 11 ligand PET imaging in patients with biochemical recurrence after radical prostatectomy: diagnostic performance and impact on therapeutic decision making. Eur J Nucl Med Mol Imaging. 2018;45:235-242.

34. Tendulkar RD, Agrawal S, Gao T, et al. Contemporary update of a multi-institutional predictive nomogram for salvage radiotherapy after radical prostatectomy. J Clin Oncol. 2016;34:3648-3654.

35. King CR. Adjuvant versus salvage radiotherapy for high-risk prostate cancer patients. Semin Radiat Oncol. 2013;23:215-221.

36. Perera M, Papa N, Christidis D, et al. Sensitivity, specificity, and predictors of positive ${ }^{68} \mathrm{Ga}$-prostate-specific membrane antigen positron emission tomography in advanced prostate cancer: a systematic review and meta-analysis. Eur Urol. 2016;70:926-937.

37. Heidenreich A, Bastian PJ, Bellmunt J, et al. EAU guidelines on prostate cancer. Part II: treatment of advanced, relapsing, and castration-resistant prostate cancer. Eur Urol. 2014;65:467-479.

38. Einspieler I, Rauscher I, Düwel C, et al. Detection efficacy of hybrid ${ }^{68} \mathrm{Ga}$-PSMA ligand PET/CT in prostate cancer patients with biochemical recurrence after primary radiation therapy defined by Phoenix criteria. J Nucl Med. 2017;58: 1081-1087.

39. Valicenti RK, Thompson I Jr, Albertsen P, et al. Adjuvant and salvage radiation therapy after prostatectomy: American Society for Radiation Oncology/American Urological Association guidelines. Int J Radiat Oncol Biol Phys. 2013;86: 822-828.

40. Ciezki JP, Klein EA, Angermeier K, et al. A retrospective comparison of androgen deprivation (AD) vs. no $\mathrm{AD}$ among low-risk and intermediate-risk prostate cancer patients treated with brachytherapy, external beam radiotherapy, or radical prostatectomy. Int J Radiat Oncol Biol Phys. 2004;60:1347-1350.
41. Buchan NC, Goldenberg SL. Intermittent versus continuous androgen suppression therapy: do we have consensus yet? Curr Oncol. 2010;17(suppl):S45-S48.

42. Furuya Y, Akakura K, Akimoto S, Inomiya H, Ito H. Pattern of progression and survival in hormonally treated metastatic prostate cancer. Int J Urol. 1999;6:240244.

43. Tree AC, Khoo VS, Eeles RA, et al. Stereotactic body radiotherapy for oligometastases. Lancet Oncol. 2013;14:e28-e37.

44. Ost P, Bossi A, Decaestecker K, et al. Metastasis-directed therapy of regional and distant recurrences after curative treatment of prostate cancer: a systematic review of the literature. Eur Urol. 2015;67:852-863.

45. Henkenberens C, von Klot CA, Ross TL, et al. ${ }^{68}$ Ga-PSMA ligand PET/CTbased radiotherapy for lymph node relapse of prostate cancer after primary therapy delays initiation of systemic therapy. Anticancer Res. 2017;37:12731279 .

46. Dietlein F, Kobe C, Neubauer S, et al. PSA-stratified performance of ${ }^{18} \mathrm{~F}$ - and ${ }^{68} \mathrm{Ga}$ PSMA PET in patients with biochemical recurrence of prostate cancer. J Nucl Med. 2017;58:947-952.

47. Dietlein M, Kobe C, Kuhnert G, et al. Comparison of $\left[{ }^{18} \mathrm{~F}\right] \mathrm{DCFPyL}$ and $\left[{ }^{68} \mathrm{Ga}\right]$ Ga-PSMA-HBED-CC for PSMA-PET imaging in patients with relapsed prostate cancer. Mol Imaging Biol. 2015;17:575-584.

48. Giesel FL, Knorr K, Spohn F, et al. Detection efficacy of ${ }^{18}$ F-PSMA-1007 PET/ $\mathrm{CT}$ in 251 patients with biochemical recurrence of prostate cancer after radical prostatectomy. J Nucl Med. 2019;60:362-368.

49. Andriole G, Kostakoglu L, Chau A, et al. The impact of positron emission tomography with ${ }^{18} \mathrm{~F}$-fluciclovine on the management of patients with biochemical recurrence of prostate cancer: results from the LOCATE trial. J Urol.2019; 201:322-331.

50. Gorin MA, Pienta KJ, Pomper MG, et al. Prostate cancer local recurrence detected with both ${ }^{18} \mathrm{~F}$-fluciclovine and PSMA-targeted ${ }^{18} \mathrm{~F}$-DCFPyL PET/CT. Urology. 2017;107:9-10.

51. Zschaeck S, Wust P, Beck M, et al. Intermediate-term outcome after PSMA-PET guided high-dose radiotherapy of recurrent high-risk prostate cancer patients. Radiat Oncol. 2017;12:140.

52. Emmett L, Van Leeuwen P, Nandurkar R, et al. Treatment outcomes from ${ }^{68}$ GaPSMA PET CT informed salvage radiation treatment in men with rising PSA following radical prostatectomy: prognostic value of a negative PSMA PET. J Nucl Med. 2017;58:1972.

53. Schmidt-Hegemann NS, Fendler WP, Ilhan H, et al. Outcome after PSMA PET/ CT based radiotherapy in patients with biochemical persistence or recurrence after radical prostatectomy. Radiat Oncol. 2018;13:37.

54. Emmett L, Metser U, Bauman G, et al. A prospective, multi-site, international comparison of F-18 fluoro-methyl-choline, multi-parametric magnetic resonance and Ga-68 HBED-CC (PSMA-11) in men with high-risk features and biochemical failure after radical prostatectomy: clinical performance and patient outcomes. J Nucl Med. November 15, 2018 [Epub ahead of print].

55. Schmidt-Hegemann NS, Stief C, Kim TH, et al. Outcome after PSMA PET/CT based salvage radiotherapy in patients with biochemical recurrence after radical prostatectomy: a bi-institutional retrospective analysis. J Nucl Med. 2018; Epub ahead of print. 